\title{
Detection and extraction of heavy metal ions using paper-based analytical devices fabricated via atom stamp printing
}

Yanfang Guan ${ }^{1}$ and Baichuan Sun ${ }^{1}$

\begin{abstract}
As a promising concept, microfluidic paper-based analytical devices ( $\mu$ PADs) have seen rapid development in recent years. In this study, a new method of fabricating MPADs by atom stamp printing (ASP) is proposed and studied. The advantages of this new method compared to other methods include its low cost, ease of operation, high production efficiency, and high resolution (the minimum widths of the hydrophilic channels and hydrophobic barriers are 328 and $312 \mu \mathrm{m}$, respectively). As a proof of concept, $\mu$ PADs fabricated with the ASP method were used to detect different concentrations of $\mathrm{Cu}^{2+}$ via a colorimetric method. Moreover, combined with a distance-based detection method, these devices achieved a $\mathrm{Cu}^{2+}$ detection limit of down to $1 \mathrm{mg} / \mathrm{L}$. In addition, a new paper-based solid-liquid extraction device (PSED) based on a three-dimensional (3D) MPAD with a "3 +2 " structure and a recyclable extraction mode was developed. Specifically, using the characteristics of paper filtration and capillary force, the device completed multiple extraction and filtration steps from traditional solid-liquid extraction processes with high efficiency. The developed PSED platform allows the detection of heavy metal ions much more cheaply and simply and with a faster response time at the point of care, and it has great promise for applications in food safety and environmental pollution in resource-limited areas.
\end{abstract}

\section{Introduction}

In the 1990s, the concept of a "lab on a chip (LOC)" was proposed based on the development of microfluidic technology ${ }^{1,2}$. The main feature of this technology is the ability to perform biochemical analysis on a chip that is a few square centimeters or smaller and to realize sample mixing, purification, separation, and other operations, thus saving on both space and cost. Microfluidic paperbased analytical devices ( $\mu$ PADs) were first proposed by Martinez et al. $^{3}$ as a device to replace traditional microfluidic chips, such as chips based on glass ${ }^{4,5}$ and silicon ${ }^{6,7}$, with the advantages of simple fabrication, low cost, portability, and disposability, and they have widespread applications in the point-of-care testing (POCT) field ${ }^{8}$.

The principle of fabricating $\mu$ PADs is to hydrophobically treat paper substrates to form distinct

\footnotetext{
Correspondence: Yanfang Guan (yguan@haut.edu.cn)

${ }^{1}$ School of Electromechanical Engineering, Henan University of technology, Zhengzhou 450001, China
}

hydrophobic and hydrophilic areas, thus restricting and guiding fluid flow. At present, there are a number of methods that can be used to fabricate $\mu$ PADs, such as photolithography ${ }^{3,9}$, wax printing $^{10-14}$, paper cutting ${ }^{15}$, drawing $^{16}$, inkjet printing ${ }^{17-19}$, laser cutting ${ }^{20}$, and stamping $^{21-26}$. For the first time, Martinez et al. ${ }^{3}$ used photolithography technology with a SU-8 photoresist to fabricate a hydrophobic pattern on paper. He et al. ${ }^{9}$ fabricated $\mu$ PADs with high resolution (hydrophilic channel was $233 \pm 30 \mu \mathrm{m}$ wide) using filter paper that was preprocessed with octadecyl trichlorosilane solution and deep ultraviolet light.

To achieve rapid and inexpensive production, $\mathrm{Lu}$ et al. ${ }^{10,11}$ and Carrilho et al. ${ }^{12}$ produced $\mu$ PADs by wax printing. The process of patterning paper with wax is quite simple, and only a commercial wax printing machine and a heating board or oven are needed in device production, which can be conducted at home. If $\mu$ PADs with high resolution are not needed in the experiment, simple paper cutting ${ }^{15}$ and 
drawing ${ }^{16}$ can be used for their production, but the production efficiency is lower than that of other methods. In 2008, Abe et al. ${ }^{17}$ completed the fabrication of $\mu$ PADs by inkjet printing with "chemical sensing inks" and achieved a relatively high channel accuracy of $550 \mu \mathrm{m}$. Up to now, laser cutting has been the best way to achieve the highest accuracy of channel resolution on paper, which can reach $62 \pm 1 \mu \mathrm{m}$ and can be used on any paper with a hydrophobic surface coating $^{20}$, such as wax paper or parchment paper; however, this operation is more complex, and the paper needs further chemical treatment.

Stamping, as another production technology, has been widely used, and different types of stamps can be used, such as a stamp with paper and $\operatorname{tape}^{21}$, a polydimethylsiloxane (PDMS) high-relief stamp ${ }^{22}$, an iron ${ }^{23}$ or steel stamp $^{24}$, and a flash foam stamp (FFS $)^{25,26}$. The hydrophobic material is printed on paper by the stamps, which are designed to be the desired pattern. Most of the above stamps are hard stamps, which are difficult to make and have the same disadvantages as wax printing, such as low resolution, because wax is commonly used as the hydrophobic solvent. An FFS overcomes these shortcomings, realizing a relatively high resolution using PDMS as the hydrophobic solvent and has the advantages of simple operation and low cost $\mathrm{t}^{25}$. However, it takes a long time to prepare, especially when the stamps are immersed in PDMS solvent, which takes more than half an hour for complete production. In addition, the stamp can only be printed several times after each soaking and must be resoaked, which takes a certain amount of time. Therefore, when considering the type of stamp to use, it is necessary to consider the cost of time while utilizing the economic benefits and convenience.

Atom stamps (ASs), also called machine-engraved penetrating stamps, can be manually engraved or can be made by a laser engraving machine and have the advantages of low cost, high efficiency, and high resolution. In situations where high resolution is not needed for $\mu$ PADs, it is possible to carve the stamp pad manually. While manual production saves on cost, it requires practice and skill. A laser engraving machine works with common drawing software, such as AutoCAD and CorelDRAW, to create pattern designs; therefore, an AS is also known as a laser engraving seal stamp. Printing can be performed with an AS because the stamps can absorb ink due to their microporous structure, and the process is simple. In this study, an AS was used as a new method to produce $\mu$ PADs, termed ASP (AS printing). For $\mu$ PAD production, a stamp of the required pattern needs to be soaked in PDMS solvent, then printed on paper, and left in a vacuum drying box or oven for a moment to complete the entire fabrication process.

To date, there have been many methods to detect heavy metal ions based on $\mu \mathrm{PADs}^{27}$, such as colorimetric detection $^{28-30}$, fluorescence detection ${ }^{31}$, and electrochemical detection ${ }^{32}$. Xu et al..$^{28}$ simultaneously detected iron and nickel ions by a colorimetric method through "multichannel" $\mu$ PADs, which proved the effectiveness of $\mu$ PADs in detecting heavy metal ions. Fu et al. ${ }^{31}$ made a new paper-based sensor using the fluorescence quenching function of gold nanoparticles (Au NPs), and the detection procedure was completed in $15 \mathrm{~min}$. Hu et al. ${ }^{32}$ used paper-based capacitive sensors (PCSs) to realize chemical identification and quantitative detection, including the quantitative detection of heavy metal ions; this approach integrated electrochemical methods and paper-based methods to eliminate the need for large-scale equipment in the detection procedure, making it more environmentally friendly. Among these three methods, the colorimetric method is the most commonly used because it is simple, is easy to operate, and does not require complex equipment to meet the basic needs of on-site detection. However, it is difficult to achieve accurate quantitative detection with the colorimetric method, and there is low accuracy with this method. Electrochemical detection overcomes the problem of low accuracy and can achieve quantitative detection, but it is relatively difficult to perform and requires relatively expensive testing equipment. Fluorescence detection can achieve targeted detection with high accuracy and reduce some necessary interference; however, it needs special detection instruments or reagents, and it is challenging to use. In this study, we chose the colorimetric method to detect $\mathrm{Cu}^{2+}$.

As a proof of concept, $\mu$ PADs fabricated via ASP were used to perform semiquantitative detection of $\mathrm{Cu}^{2+}$ by a colorimetric method ${ }^{33}$. Alternatively, distance-based detection can be used for detection instead of the colorimetric method $^{34-36}$, and it is an effective quantitative method with better accuracy. The distance-based method was first proposed by Cate et al. ${ }^{35}$, and they demonstrated the method on $\mu$ PADs for the detection of glucose, nickel, and so on. Later, Pratiwi et al. ${ }^{36}$ used a porphyrin derivative to detect copper ions using the distance-based method, and the minimum detection limit achieved was $1 \mathrm{mg} / \mathrm{L}$. In this study, we combine the colorimetric method with the distance-based method to achieve quantitative detection of the $\mathrm{Cu}^{2+}$ concentration.

To further demonstrate the versatility of our $\mu$ PADs, we introduced an integrated device, which combined 3D $\mu$ PADs and a homemade micropump to achieve integrated extraction and filtration, called a paper-based soil-liquid extraction device (PSED). Usually, the solid-liquid extraction process of heavy metal ions in soil includes centrifugation, oscillation, and more $^{37}$, which require specialized equipment such as a centrifuge ${ }^{38}$ and ultrasonic vibration ${ }^{39}$. Therefore, the traditional process does not match the principles of low cost, portability, and miniaturization in the POCT field ${ }^{40}$, and it is necessary to 
develop a cheap and simple extraction device to meet the needs of on-site detection in resource-limited areas. The PSED studied here capitalizes on the advantages of paper itself, such as its low cost, portability, and filterability, and demonstrated superior performance when used as an extraction device in our experiment.

\section{Results and discussion}

\section{Resolution analysis of $\mu$ PADs by the ASP method}

Resolution is an important metric to evaluate the performance of $\mu$ PADs. Resolution refers to the minimum channel width of the fluid flow passages on the paper and the minimum width of the hydrophobic barrier that prevents the flow of fluid. The structure of the hydrophilic channels $(0.3-1.3 \mathrm{~mm})$ and hydrophobic barriers $(0.2-1.1 \mathrm{~mm})$ was designed as shown in Fig. 1a, b. To observe the flow through the hydrophilic channels and the hydrophobic barriers accurately, a blue dye was added to the channels. As shown in Fig. 1c, d, it was obvious that when the width of the hydrophilic channel was $0.4 \mathrm{~mm}$, the liquid could flow normally, and when the hydrophobic barrier was $0.2 \mathrm{~mm}$, the flow was blocked. As seen in Fig. 1e, $f$, the theoretical widths of the hydrophilic channels and hydrophobic barriers are different from their actual widths in the $\mu$ PADs.

The actual widths of the hydrophilic channel were generally smaller than the theoretical widths; on the contrary, the actual widths were generally larger than the theoretical widths for the hydrophobic barriers according to the measurement results and the fitting line. This phenomenon is due to the diffusion and permeation of PDMS that occurred during the printing of the hydrophobic materials on the filter paper by AS fabrication. Furthermore, there is an approximately proportional relationship between the actual value and the theoretical value, and the correlation coefficient $\left(R^{2}\right)$ is $\sim 0.9965$ for the hydrophilic channels and 0.999 for the hydrophobic barriers. The actual minimum widths of the hydrophilic channels and hydrophobic barriers measured under a microscope were 328 and $312 \mu \mathrm{m}$ (Fig. 1e, f), respectively.

Among $\mu$ PADs made by different methods, $\mu$ PADs made by laser cutting can achieve the highest resolution, with a minimum hydrophilic channel width of $62 \pm$ $1 \mu \mathrm{m}^{20}$. However, special paper materials are needed, the cost is high, and the operation is complex. Drawing and cutting have low costs and are easy to operate, while the

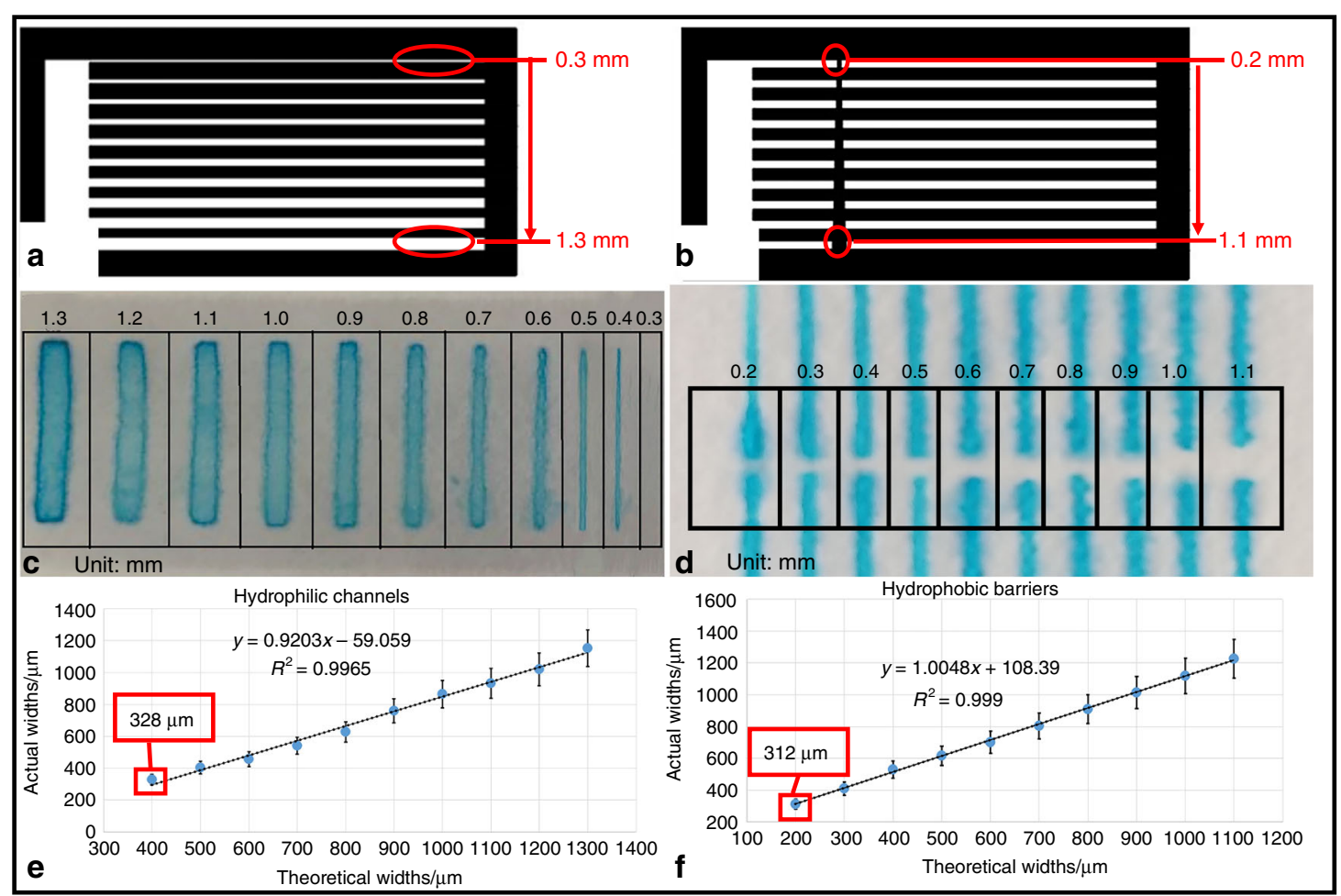

Fig. 1 Resolution testing of $\mu$ PADs made by the ASP method. a Structural picture of the hydrophilic channels. $\mathbf{b}$ Structural picture of the hydrophobic barriers. $\mathbf{c}$ Hydrophilic channels tested with blue dye. $\mathbf{d}$ Hydrophobic barriers tested with blue dye. e Comparison of the theoretical and actual widths of the hydrophilic channels. $\mathbf{f}$ Comparison of the theoretical and actual widths of the hydrophobic barriers. 
Table 1 Comparison of the $\mu$ PAD fabrication methods.

\begin{tabular}{|c|c|c|c|c|}
\hline Method & $\begin{array}{l}\text { Channel } \\
(\mu \mathrm{m})\end{array}$ & $\begin{array}{l}\text { Barrier } \\
(\mu \mathrm{m})\end{array}$ & Advantages & Disadvantages \\
\hline Photolithography ${ }^{3,9}$ & $233 \pm 30$ & $137 \pm 21$ & High resolution & Complex operation; high cost \\
\hline Wax printing ${ }^{12}$ & $\sim 600$ & $\sim 1300$ & Easy to fabricate, low cost & Low resolution \\
\hline Inkjet printing ${ }^{17}$ & 550 & 302 & High resolution, rapid & Special printers \\
\hline Cutting $^{15}$ & $\sim 2000$ & - & Low cost, easy to operate & Low resolution \\
\hline Laser cutting ${ }^{20}$ & $62 \pm 1$ & 80 & High resolution & $\begin{array}{l}\text { Complex operation, special paper materials } \\
\text { are needed }\end{array}$ \\
\hline $\mathrm{FFSL}^{25}$ & $632 \pm 27$ & $306 \pm 20$ & Low cost, easy to fabricate & Low resolution \\
\hline ASP & 328 & 312 & $\begin{array}{l}\text { Low cost, rapid, high resolution, easy to } \\
\text { fabricate }\end{array}$ & \\
\hline
\end{tabular}

Table 2 Cost comparison of the ASP and FFSL methods.

\begin{tabular}{llll}
\hline Materials & Amount & Cost of FFSL & Cost of ASP \\
\hline Filter paper & $40 \times 40 \mathrm{~mm}^{2}$ & $¥ 0.15$ & $¥ 0.15$ \\
Atom stamp & $40 \times 40 \mathrm{~mm}^{2}$ & - & $¥ 0.03$ \\
PDMS & $0.5 \mathrm{~g}$ & $¥ 0.4$ & $¥ 0.4$ \\
Flash foam & $40 \times 40 \mathrm{~mm}^{2}$ & $¥ 0.09$ & - \\
Tracing paper & $40 \times 40 \mathrm{~mm}^{2}$ & $¥ 0.001$ & - \\
Mask & 1 piece of paper & $¥ 0.01$ & - \\
Electricity & $\approx 0.1 \mathrm{kw} / \mathrm{h}$ & $¥ 0.01$ & $¥ 0.01$ \\
Total & & $¥ 0.91 / \$ 0.15$ & $¥ 0.59 / \$ 0.09$ \\
\hline
\end{tabular}

resolution (the width of the hydrophilic channels was $\sim 2000 \mu \mathrm{m})$ is low ${ }^{15,16}$. Wax printing $(600 \mu \mathrm{m})^{12}$ and inkjet printing $(550 \mu \mathrm{m})^{17}$ require special printers. Wax printers are seldom sold on the market, and inkjet printing requires customized printers. FFS lithography (FFSL) and ASP are stamping methods, but the resolution of the FFSL method (the widths of hydrophilic channels and hydrophobic barriers were $632 \pm 27$ and $306 \pm 20 \mu \mathrm{m}$, respectively $)^{25}$ is significantly lower than that of the ASP method. Additionally, ASP is more efficient than FFSL in the production of $\mu$ PADs. A detailed comparison of the $\mu$ PAD fabrication methods is shown in Table 1 .

\section{Economic analysis of the ASP method}

As mentioned, the advantages of the ASP method include its low cost and easy operation. The most expensive one-time cost is the laser engraving machine. Nonetheless, the laser engraving machine is highly efficient, producing $\sim 20$ stamps in a min, and is manufactured anywhere that produces seals. The total cost of the other materials is $\sim 9$ cents (¥0.59/\$0.09), as seen in
Table 2, which is lower than the cost of the FFSL method $^{25}$ (¥0.91/\$0.15). Each AS can be reused many times, which greatly reduces the cost of production.

\section{Colorimetric analysis of $\mathrm{Cu}^{2+}$ \\ Colorimetric card detection of $\mathrm{Cu}^{2+}$}

As shown in Fig. $2 \mathrm{a}-\mathrm{g}$, the $\mu$ PADs with $\mathrm{Cu}^{2+}$ clearly change from white to yellow, and the color of the yellow complexes deepens with increasing $\mathrm{Cu}^{2+}$ concentration. The image was processed by the ImageJ software, as shown in Fig. 2h, and with an increase in ion concentration, the grayscale value decreased (gray value of 255 represents white or no color, and gray value of 0 represents black or darker color), indicating that the color of the complex deepened.

\section{Distance-based detection of $\mathrm{Cu}^{2+}$}

Figure $3 \mathrm{a}$ shows the distances of $\mathrm{Cu}^{2+}$ solutions with different concentrations flowing through the channels of the $\mu$ PADs. The length of the yellow-colored band increased as the concentration of $\mathrm{Cu}^{2+}$ increased. As shown in Fig. 3b, c, the colored band length was constant above $100 \mathrm{mg} / \mathrm{L}$, making it the upper limit of the device. The minimum detection concentration of $\mathrm{Cu}^{2+}$ was $1 \mathrm{mg} / \mathrm{L}$, in accordance with the World Health Organization and United States Environmental Protection Agency regulations for the maximum contamination concentration of $\mathrm{Cu}^{2+}$ in drinking water, which are 2 and $1.3 \mathrm{mg} / \mathrm{L}^{41,42}$, respectively.

\section{Heavy metal ion extraction in soil}

The working principles of a PSED are shown in Fig. 4a-c. Two main steps occur during extraction: the micropump driving ${ }^{43}$ step, which enables the device to achieve the extraction cycle shown in Fig. $4 \mathrm{~b}$, and the extraction step by the $3 \mathrm{D} \mu \mathrm{PAD}$, which utilizes the microporous characteristics of the filter paper itself to 
complete the solid-liquid extraction and filtration processes, as shown in Fig. 4a, c. First, the soil samples were stored on top of the 3D $\mu$ PAD. Then, the extraction solvent flowed out from the outlet pipe of the micropump, mixed with the soil, and flushed down into the reservoir. Concomitantly, the extraction solvent solubilized the

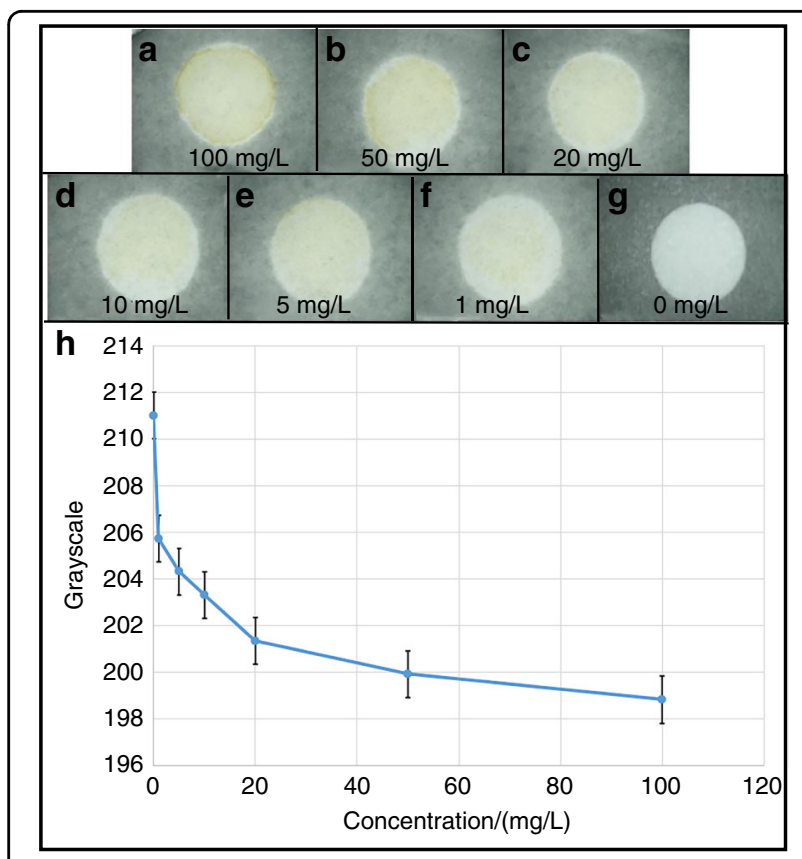

Fig. 2 Pictures of the colorimetric detection of $\mathrm{Cu}^{2+} \cdot \mathbf{a}-\mathrm{g}$ Color rendering effect of copper ions and DDTC at different concentrations. $\mathbf{h}$ Grayscale trend with the concentration of $\mathrm{Cu}^{2+}$. heavy metal ions, sucked them through the inlet pipe, and was pumped out again, forming the extraction cycle. Finally, the heavy metal ions, such as $\mathrm{Cu}, \mathrm{Zn}, \mathrm{Cd}$, and $\mathrm{Pb}$, were extracted from the soil samples via the continuous supply and pump cycle of the micropump.

As seen from Fig. $4 \mathrm{~d}-\mathrm{f}$, the concentration of the heavy metal ions $(\mathrm{Pb}, \mathrm{Cd}, \mathrm{Cu}, \mathrm{Zn}$, etc.) obtained by the PSED extraction procedure was almost the same as that obtained by traditional methods, which proves that the PSED was effective. The error rate between the PSED extraction and traditional extraction process was between 0.02 and 0.05 , which is acceptable in extraction process (the error rate $=\mid$ heavy metal ion concentration obtained by PSED extraction-heavy metal ion concentration obtained by traditional extraction//heavy metal ion concentration obtained by traditional extraction). When the error rate is $<0.05$, a method is qualified for extraction ${ }^{44}$. In the experiment, solid-liquid ratios of 1:5, 1:10, 1:15, and 1:20 were adopted for the extraction process (Fig. 4g), and under the condition of satisfying the extraction accuracy, the solid-liquid ratio of 1:10 completed the detection. By contrast, the error rates of the ratios of 1:15 and 1:20 (0.06 and 0.09, respectively) were larger than that of the ratio of $1: 10$, and therefore, these ratios were not recommended for use in extractions.

The extractant volume should not be too small for the extraction process. First, there will be losses of the extractant during the extraction process, which will remain in the $\mu$ PADs, soil, or devices. Second, if there is too little final extraction solvent, detection will be difficult. Therefore, more than $30 \mathrm{~mL}$ of the extractant is required. This

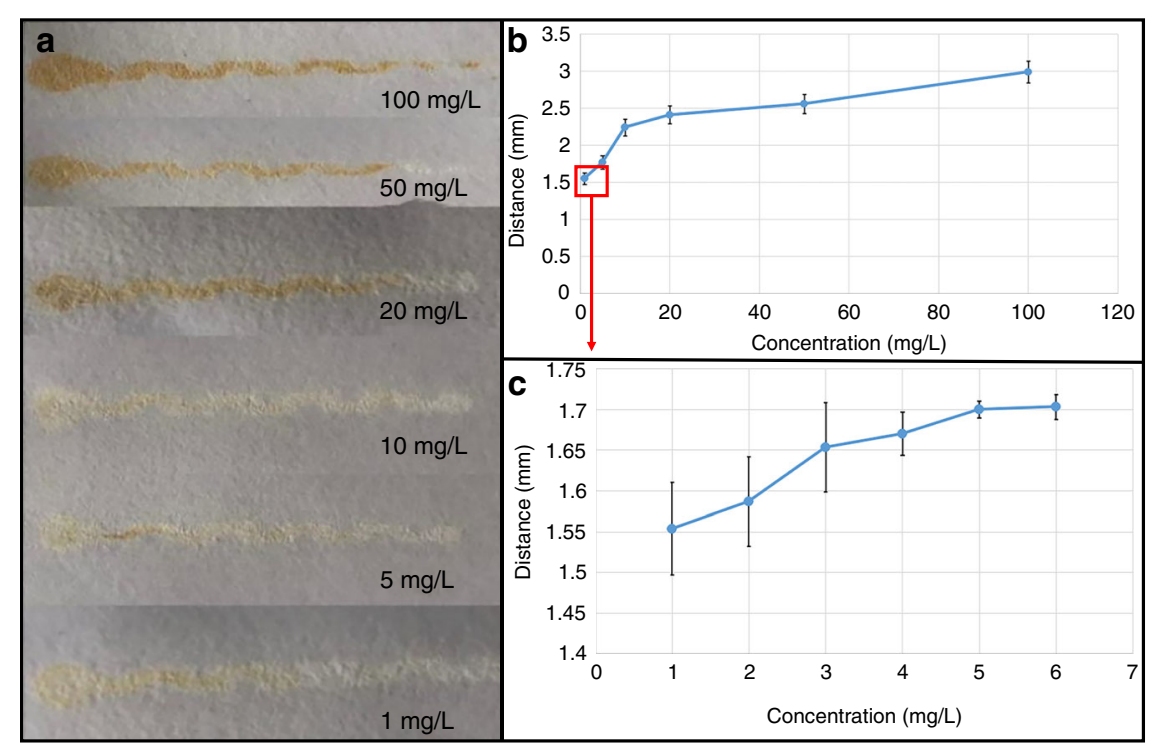

Fig. 3 Testing of the $\mathrm{Cu}^{2+}$ concentration based on the distance-based detection method. a The flow of the solution on the channel with increasing $\mathrm{Cu}^{2+}$ concentration. $\mathbf{b}$ Linear relationship between $\mathrm{Cu}^{2+}$ concentration $(0-100 \mathrm{mg} / \mathrm{L})$ and flow distance in the channel. $\mathbf{c}$ Flow length at $1-6 \mathrm{mg} / \mathrm{LCu}^{2+}$ concentrations. 


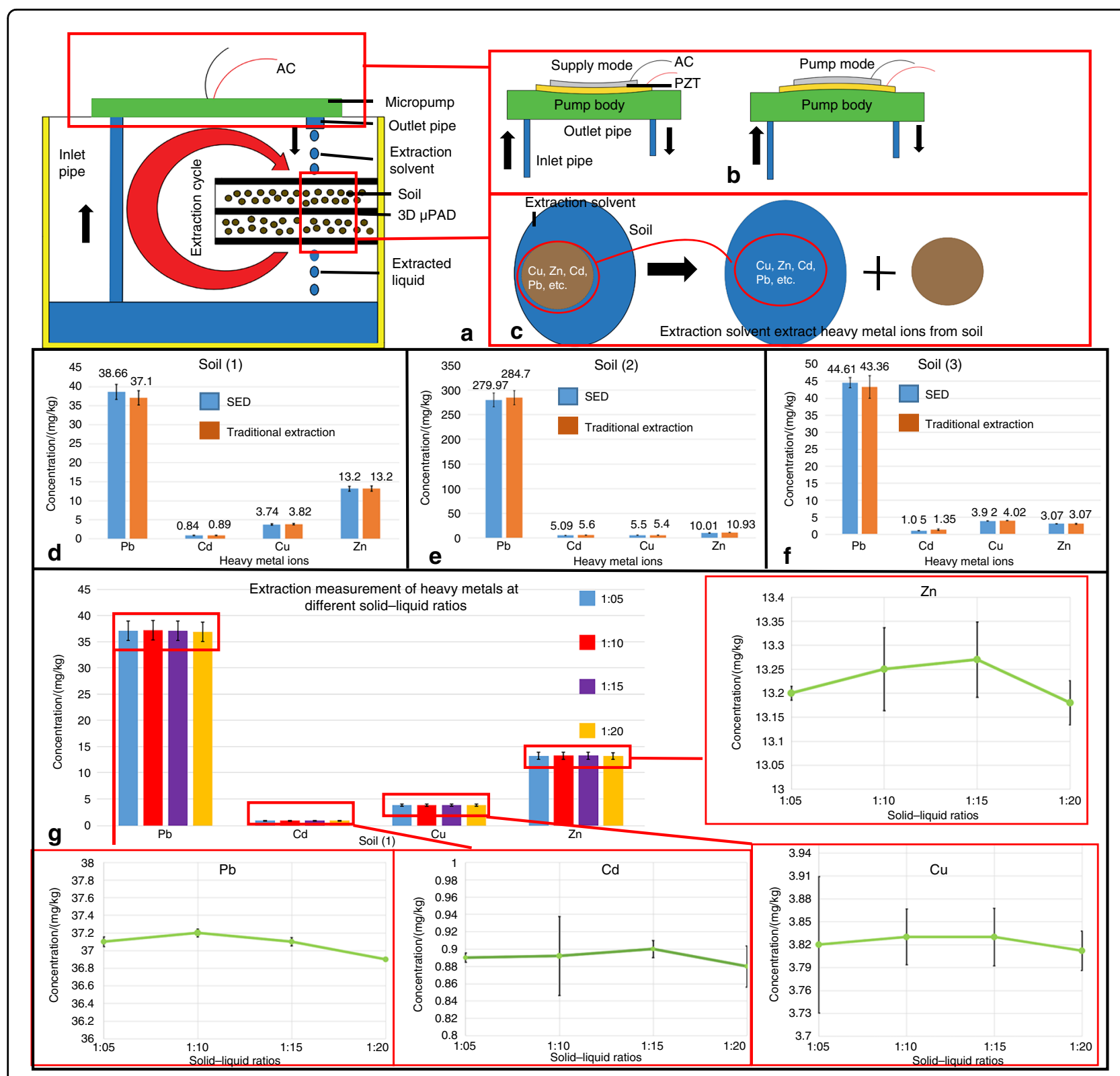

Fig. 4 Working principle and comparison of detected heavy metal ion concentrations between the PSED and traditional extraction method. a-c Schematic of the working principle of the PSED. $\mathbf{d}-\mathbf{f}$ The concentration results of the heavy metal ions in soils (1), (2), and (3), respectively. $\mathbf{g}$ Comparison of the heavy metal ion concentrations obtained by PSED extraction at different solid-liquid ratios.

experiment used $40 \mathrm{~mL}$ of extractant and $4 \mathrm{~g}$ of soil, and the solid-liquid ratio was 1:10. To ensure complete extraction, we also compared the extraction time and found that $20 \mathrm{~min}$ was sufficient for complete extraction of the heavy metal ions. Each $3 \mathrm{D} \mu \mathrm{PAD}$ can hold $2 \mathrm{~g}$ of soil, and therefore, the extraction procedure takes $40 \mathrm{~min}$ to complete.

Compared with the traditional extraction method, 3D $\mu \mathrm{PAD}$ extraction omitted the process of filtration, which makes the operation simpler and the extraction accuracy higher. Moreover, 3D $\mu$ PADs have the advantages of being portable and cheap, and removing the need for large equipment, which makes extraction simple. It is worth noting that the size of the device can be adjusted according to different needs, providing more flexibility for real-world applications.

\section{Conclusions}

$\mu$ PADs, as a new type of detection device, have been used in many fields, such as environmental monitoring and food safety. The fabrication technology of $\mu$ PADs has become a 
focus of research in the past few years. In this study, a new $\mu \mathrm{PAD}$ fabrication method using ASP was proposed. The $\mu$ PADs fabricated with ASP achieved high resolution, $328 \mu \mathrm{m}$ for the hydrophilic channels and $312 \mu \mathrm{m}$ for the hydrophobic barriers. Compared with other methods, ASP is low cost and has simple operation, short preparation time, high resolution, and higher sensitivity than other methods (wax printing, inkjet printing, photolithography method, etc.). Moreover, this method can be used in most situation because PDMS is a transparent liquid, avoiding the possible contamination of the hydrophobic channels by the hydrophobic agent. We demonstrated that ASPfabricated $\mu$ PADs can detect $\mathrm{Cu}^{2+}$ using a colorimetric method and that, combined with a distance-based detection method, the approach can achieve the detection of $\mathrm{Cu}^{2+}$ at a concentration of $1 \mathrm{mg} / \mathrm{L}$.

In addition, a new solid-liquid extraction device, PSED, was proposed to extract heavy metal ions from the soil. This device integrated the extraction, filtration, and collection processes and requires fewer experimental samples to meet the needs of POCT by reducing sample loss. In addition, the whole device has high extraction efficiency, low cost, and no pollution, and it can be improved according to its own requirements and can meet the demands of most solid-liquid extraction processes. This device is also not limited to soil. Moreover, the device is simple to manufacture and can be produced with low cost (3D printing). Therefore, it is suitable for use in areas with no professional equipment and low income and can be adjusted according to extraction needs. However, the simple operation, highly integrated nature, and production of real POCT products need to be improved for use of this device.

\section{Materials and methods Materials and devices}

Whatman No. 1, No. 4, and No. 5 filter papers were purchased from Whatman Company, UK. PDMS (Sylgard 184) was purchased from Dow Corning, USA. Standard copper solution $(1.0 \mathrm{~g} / \mathrm{L})$ was purchased from National Standard Substances Research Center, China. Ammonia and copper reagents were purchased from Brilliant, China. DTPA (diethylenetriamine pentaacetic acid) extraction solution was made in the Chemistry Research Laboratory of Henan University of Technology. Deionized water was homemade. Soil samples were collected from three different areas: No. 1 (Lingshan Village of Jiyuan, China), No. 2 (smelting plant in Zhengzhou, China), and No. 3 (Tangshi greenhouse, China). The laser engraving machine was purchased from Laser Star, China. The atomic absorption spectrometer was purchased from Thermo Fisher, USA. The fused deposition molding (FDM) 3D printer (D-Force 400) was purchased from Triangle Laboratory Co., Ltd. (Jiangsu, China). The power amplifier and the micropump were homemade.

\section{Paper selection}

The first step to make $\mu$ PADs is choosing the right type of paper. In this experiment, three types of filter paper were selected for comparison: Whatman No. 1, No. 4, and No. 5 filter papers. Among them, No. 1 filter paper has the advantages of moderate pore size and moderate flow rate and is easy to observe and detect; therefore, it is often used to make $\mu \mathrm{PADs}^{45}$. No. 4 filter paper, because of its large aperture, has a faster flow rate on the paper, and it is not easy to observe the fluid's flow and reaction. Moreover, it is thicker than No. 1 filter paper, making it difficult for the hydrophobic agent to penetrate its interior. Fluid flows slowly on No. 5 filter paper since the pore size is small, which is also not easy for hydrophobic solvents to penetrate and makes it hard to perform paper bonding. Therefore, No. 1 filter paper was employed as the paper base in the experiment and production process. The papers' parameters are listed in Table 3.

\section{Fabrication of the AS}

The AS is made of a special sponge material with a microporous structure, which is divided into a reservoir layer (storing ink) and a lithographic layer (engraving pattern), as shown in Fig. 5a. The different structures of the reservoir layer and lithographic layer are shown in Fig. 5b-g, respectively. As shown in Fig. 5b-g, both the surface and the interior of the AS have microporous structures, and the pores on the surface, which can easily form the microstructure, are smaller than those in the reservoir layer, which can well absorb the hydrophobic solvent.

To avoid burning or affecting the accuracy of the AS due to overheating durin the carving process, it is necessary to immerse the stamp in water for 2-3 min prior to carving. Then, the pattern designed by AutoCAD or CorelDraw software can be imported into the laser engraving machine to complete the production of the AS (Fig. 5h). During the laser engraving procedure, the spot size is $38 \mu \mathrm{m}$ (the beam width is $300 \mu \mathrm{m}$ ), and the speed is $500 \mathrm{~mm} / \mathrm{s}$. The current used in the engraving procedure is related to the engraving depth. Generally, $10 \mathrm{~mA}$ is used. If the engraving is deeper, $15 \mathrm{~mA}$ can be used. The specific operation is shown in Fig. $5 \mathrm{~h}$.

\section{Fabrication of $\mu$ PADs}

The AS (Fig. 6a), which was engraved, was immersed in the prepared PDMS solvent (PDMS and curing agent mixed in a ratio of 10:1 and put into a vacuum drying box to remove bubbles, which takes $\sim 10 \mathrm{~min}$ ) to fully absorb the hydrophobic solution (see step 3 in Fig. 5h). By varying the immersion time from 2 to $5 \mathrm{~min}$, as shown in Fig. $6 \mathrm{~b}$, we observed that clear patterns can optimally be printed on paper after 5 min of immersion. As shown in Fig. $6 \mathrm{c}-\mathrm{e}$, the filter paper treated with PDMS can achieve hydrophobicity. The contact angle between water and the 
hydrophobic surface can reach $137^{\circ}$ (Fig. 6d, e) without affecting the fluid flow in the hydrophilic regions (a contact angle $>90^{\circ}$ is regarded as hydrophobic; a contact angle $<90^{\circ}$ is considered hydrophilic).

\section{Fabrication of the colorimetric card}

The colorimetric $\mu$ PADs made with the ASP method (shown in Fig. 7a with a diameter of $10 \mathrm{~mm}$ ) were immersed in a copper reagent (sodium diethyldithiocarbamate, DDTC) in advance and then dried at room temperature for detection. The principle of making the colorimetric card is that $\mathrm{Cu}^{2+}$ reacts with DDTC to produce a brown-yellow complex under weakly alkaline conditions.

The distance-based detection $\mu \mathrm{PAD}$ also needed to be immersed in DDTC solution in advance and dried, and then different concentrations of $\mathrm{Cu}^{2+}$ solution were added to the device dropwise (the channel shape was an $\mathrm{S}$-shape, the total length was $30 \mathrm{~mm}$, the width was $3 \mathrm{~mm}$,

Table 3 Performance parameters of the different types of filter paper.

\begin{tabular}{lllll}
\hline Type & $\begin{array}{l}\text { Aperture } \\
(\boldsymbol{\mu m})\end{array}$ & Filtration speed & $\begin{array}{l}\text { Thickness } \\
(\boldsymbol{\mu m})\end{array}$ & $\begin{array}{l}\text { Basic } \\
\text { weight } \\
\left(\mathbf{g} / \mathbf{m}^{\mathbf{2}}\right)\end{array}$ \\
\hline No. 1 & 11 & Medium & 180 & 88 \\
No. 4 & $20-25$ & Fast & 205 & 96 \\
No. 5 & 2.5 & Slow & 200 & 105 \\
\hline
\end{tabular}

and there was a liquid storage area with a diameter of $5 \mathrm{~mm}$ at the entrance, as shown in Fig. 7b).

\section{Heavy metal ion extraction device in soil Fabrication of the $3 D \mu P A D$}

The principle of making 3D $\mu$ PADs was to superimpose $2 \mathrm{D} \mu \mathrm{PADs}$ to create hydrophilic channels that can flow vertically. The traditional methods of making $3 \mathrm{D} \mu \mathrm{PADs}$ include origami ${ }^{46}$, double-sided gluing ${ }^{47,48}$, and stapler binding ${ }^{49}$. Based on the vertical flow characteristics of 3D $\mu$ PADs, we designed a " $3+2$ " structure for a $3 D$ extraction $\mu \mathrm{PAD}$ (Fig. 8a-c). To prevent leakage and loosening during the experiment, three layers of $\mu$ PADs and two layers of PDMS films were packaged with plastic films, and glue was used to connect the layers. The sizes of the $\mu \mathrm{PAD}$ and PDMS films are shown in Fig. 8c. The hydrophilic region of the $\mu \mathrm{PAD}$ was the same as the hollow part of the PDMS film $\left(30 \times 20 \mathrm{~mm}^{2}\right)$, and the remaining part was the hydrophobic region. The thickness of the PDMS layer used to store soil or other samples was $3 \mathrm{~mm}$. Furthermore, the $3 \mathrm{D} \mu \mathrm{PAD}$ was roughly divided into two storage layers, each of which could hold $1 \mathrm{~g}$ of soil.

\section{Fabrication of the integrated PSED}

The PSED was divided into three main parts: the extraction and filtration section (Fig. 8a-c), the supporting frame (Fig. 8d-g), and the circulating liquid supply section (Fig. 8h-i). As shown in Fig. 8d-g, the four components of the supporting frame, the jig of the $\mu \mathrm{PAD}$, the top cover, the jig of the micropump, and the reservoir, were all made of PLA and processed by an FDM 3D
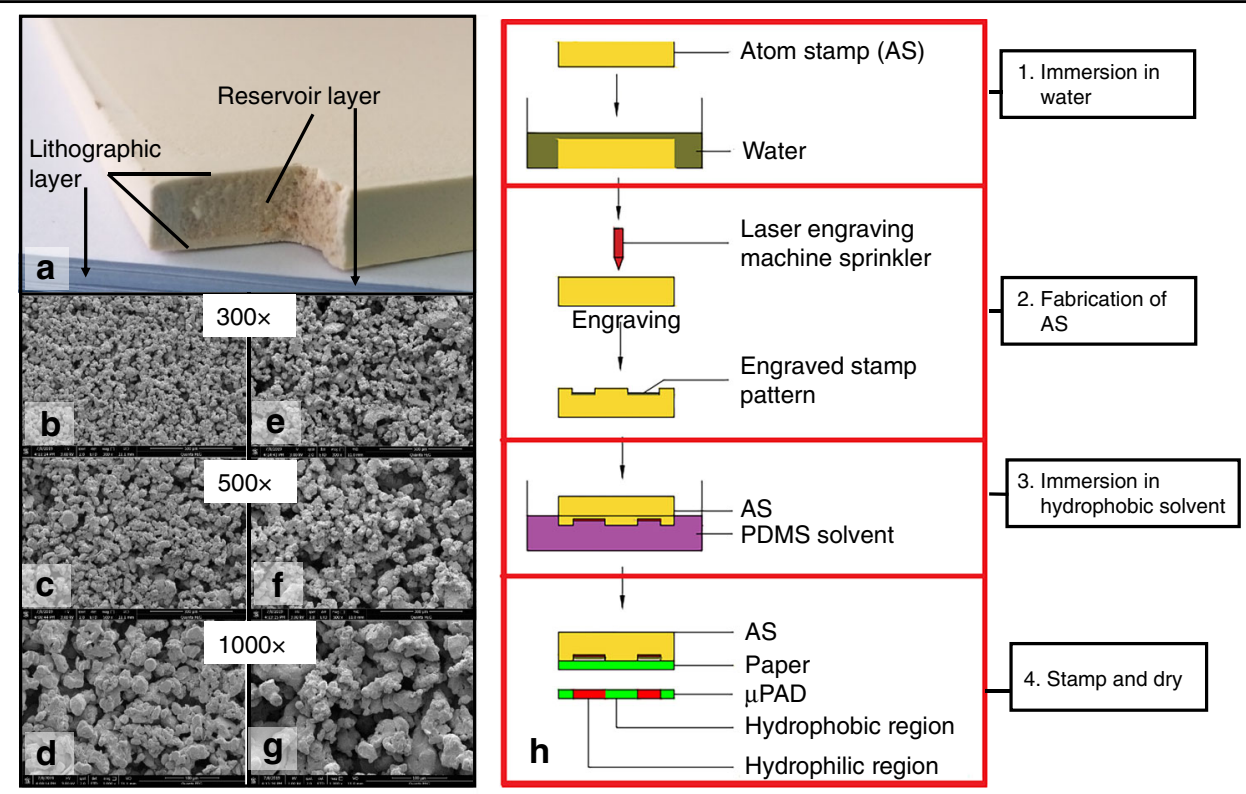

Fig. 5 Internal composition and fabrication progress of the AS. a Components of the atom stamp. $\mathbf{b}$ - $\mathbf{d}$ Microstructure of the lithographic layer. e- $\mathbf{g}$ Microstructure of the reservoir layer. $\mathbf{h}$ Fabrication process of an ASP. 


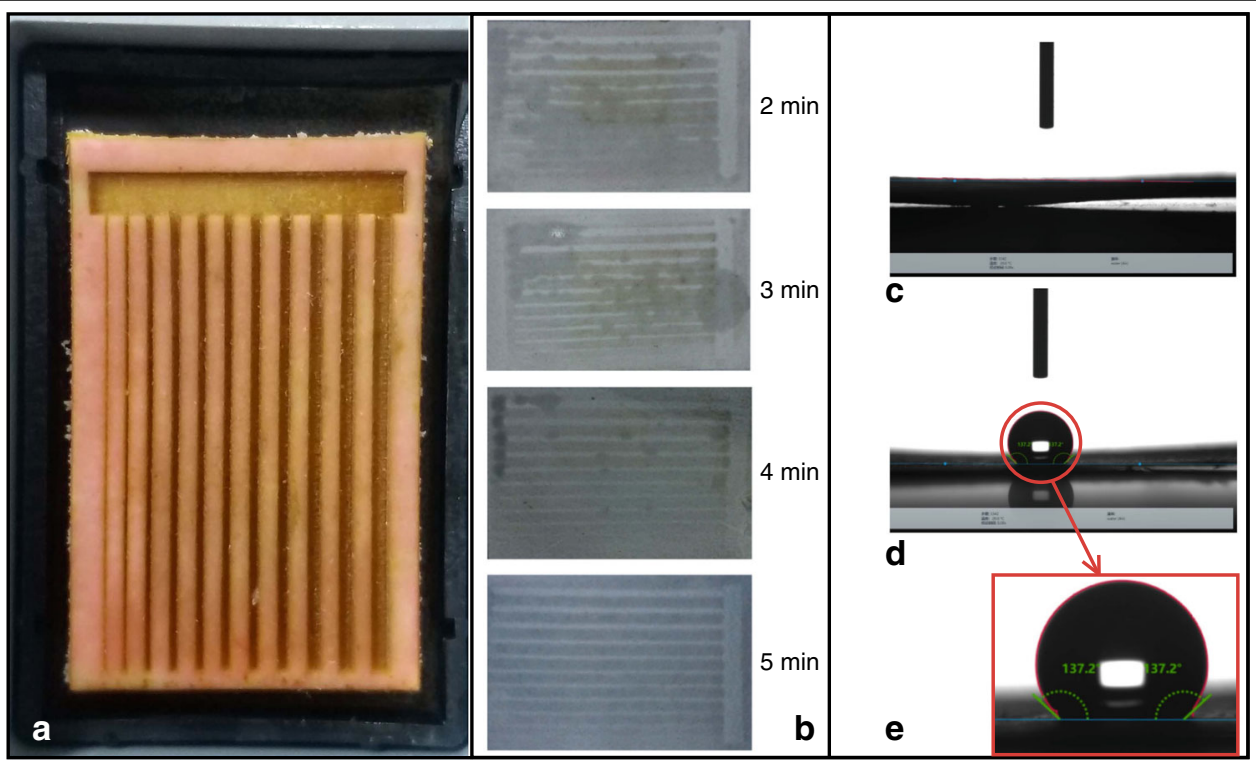

Fig. $6 \mu$ PAD fabricated by an ASP method. a AS with an engraved pattern. $\mathbf{b}$ ASs with different immersion times. $\mathbf{c}$ Contact angle between water and the hydrophilic area. $\mathbf{d}$, e Contact angle between water and the hydrophobic area.

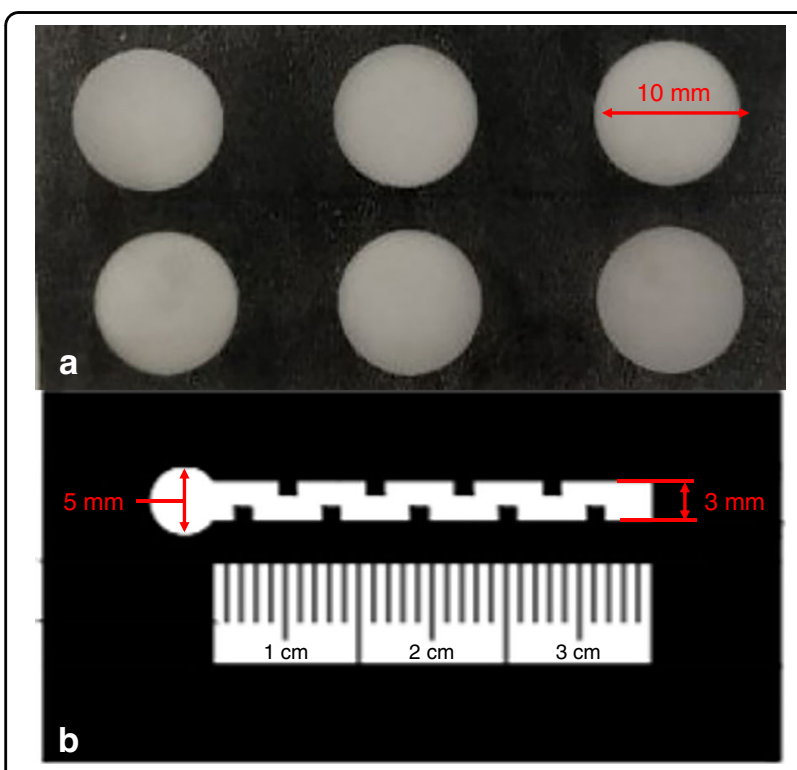

Fig. 7 Detection of $\mathrm{Cu}^{2+}$ by $\mu \mathrm{PADs}$. a Detection with a colorimetric card. $\mathbf{b}$ Detection by the distance-based method on a $\mu$ PAD.

printer. The fabricated $3 \mathrm{D} \mu \mathrm{PAD}$ was placed in the jig (Fig. 8d) and inserted into the reservoir (Fig. 8g) that had been filled with extraction solution. Then, the homemade micropump (see Fig. $8 \mathrm{~h}-\mathrm{i}$ ) was put into a groove in the top cover (Fig. 8e) to realize control of the liquid supply in the whole device. To prevent leakage of the micropump during the experiment, a jig (Fig. 8f) was used to fix the micropump, ensuring that the operation and performance of the micropump were not affected during the fixing process, and PZT (lead zirconate titanate) wires were drawn from the hole as shown in Fig. $8 \mathrm{f}$. The size of the reservoir that we designed was $50 \times 40 \times 60 \mathrm{~mm}^{3}$, and the opening of the jig of the $\mu$ PAD was $45 \mathrm{~mm}$ above the bottom; thus, it could store $40 \mathrm{~mL}$ of extraction solution (the size of the device can be improved according to different needs). The size of the jig of the $\mu \mathrm{PAD}$ was $40 \times 30$ $\mathrm{mm}^{2}$, and there were $10 \mathrm{~mm}$ holes in the wall of the reservoir so that the inlet pipe of the micropump could pass through the gap to the extraction solution.

Finally, the whole device was connected in the way shown in Fig. 8j-k to form a working PSED experimental platform. The inner structure of the PSED after packaging is shown in Fig. 8l. Since a homemade micropump was used in this experiment, a homemade power amplifier was also used for power amplification. Note that if a commercial micropump was used, only the signal generator would be needed.

The principle of extracting heavy metal ions from soil with the PSED is shown in Fig. 8m. The extraction solvent was extracted from the inlet pipe of the micropump and then added dropwise to the $3 \mathrm{D} \mu \mathrm{PAD}$ through the outlet pipe. Afterwards, the extracted liquid was infiltrated through the paper and soil by the subsequently added extraction solvent to achieve liquid circulation. The whole extraction area can be automatically wetted due to the hydrophilicity of the paper, ensuring full contact between the extraction solvent and soil. Finally, the extracted liquid was poured out, and the heavy metal ions were detected by atomic absorption spectrometry (see Fig. 8n). The experimental results were compared with those obtained with the traditional centrifugal oscillation and filtration extraction methods (Fig. 8o). 


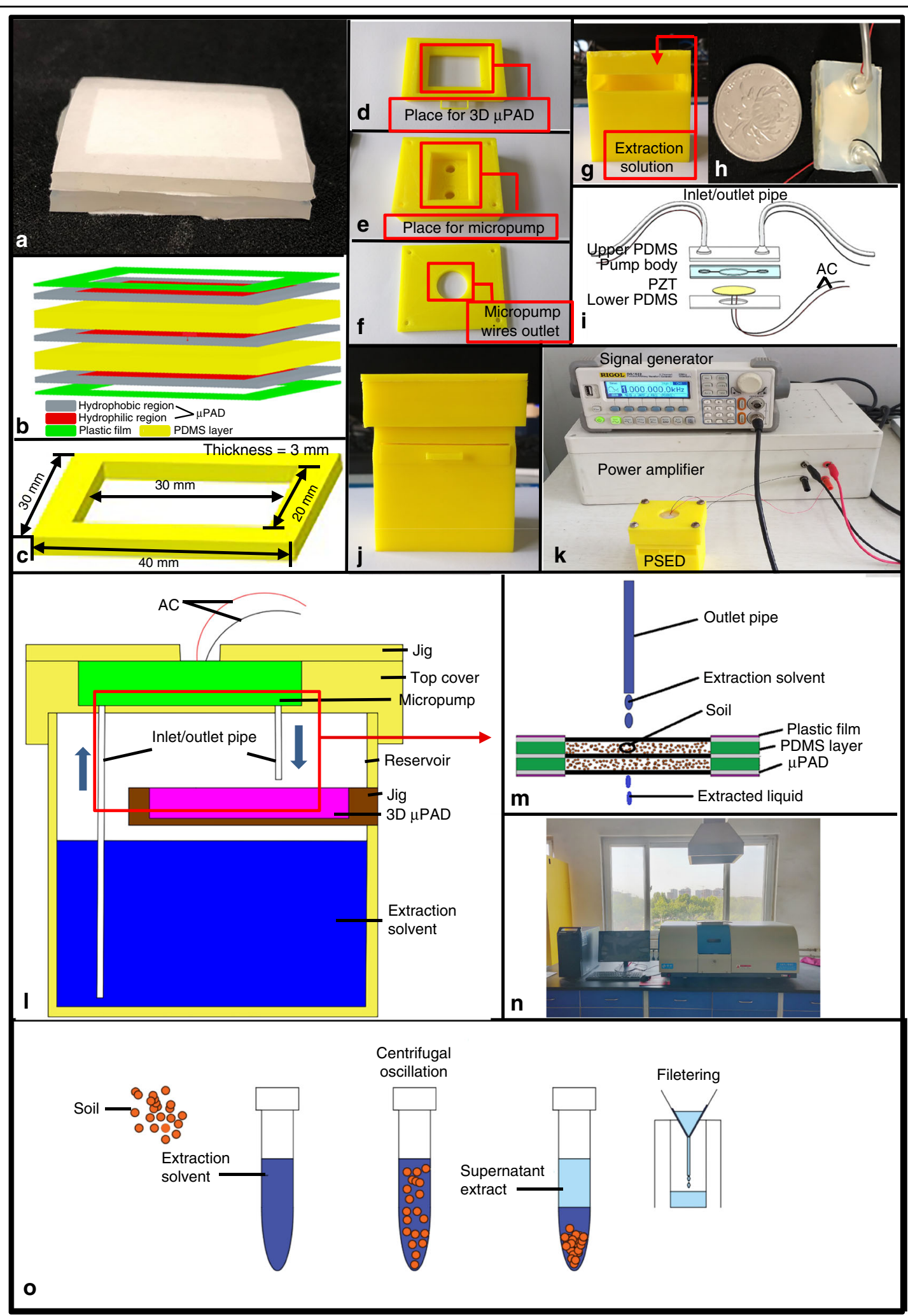

Fig. 8 Picture of an integrated soil-liquid extraction device. a Physical picture of the 3D $\mu$ PAD. $\mathbf{b}$ Structural picture of the 3D $\mu$ PAD. $\mathbf{c}$ Size of the PDMS layer. $\mathbf{d}$ Jig of the $\mu$ PAD. e Top cover. $\mathbf{f}$ Jig of the micropump. $\mathbf{g}$ Reservoir. $\mathbf{h}$ Physical picture of the micropump. $\mathbf{i}$ Composition of the micropump. $\mathbf{j}$ Assembled PSED. $\mathbf{k}$ Experimental platform of the PSED. I The inner structure of the PSED. $\mathbf{m}$ Experimental principle of the PSED. $\mathbf{n}$ Fire atomic absorption spectrometer (FASS). o Experimental principle of traditional soil-liquid extraction. 


\section{Acknowledgements}

We acknowledge professor Guangyu Liu at the School of Chemistry, Chemical Engineering and Environment, Henan University of Technology, for assistance with soil sample and extraction solvent preparation. We also thank undergraduate student Xuesen Zhang for help with the experiments. This work is sponsored by the National Natural Science Foundation of China (No. 51505128) and the Tackling Key Scientific and Technological Problems in Henan Province Fund (No. 182102410061). It is also supported by the Young Backbone Teachers Training Program of Henan University of Technology.

\section{Author contributions}

Y.G. conceived and designed the study. B.S. performed the experiments. Y.G. wrote and edited the manuscript. All authors read and approved the manuscript.

\section{Data availability}

The authors declare that the data supporting the findings of this study are available within the paper.

\section{Conflict of interest}

The authors declare that they have no conflict of interest.

Supplementary information accompanies this paper at https://doi.org/ 10.1038/s41378-019-0123-9.

Received: 26 July 2019 Revised: 24 October 2019 Accepted: 4 November 2019

Published online: 09 March 2020

\section{References}

1. Sackmann, E. K., Fulton, A. L. \& Beebe, D. J. The present and future role of microfluidics in biomedical research. Nature 507, 181-189 (2014).

2. Guo, L., Feng, J., Fang, Z., Xu, J. \& Lu, X. Application of microfluidic "lab-on-achip" for the detection of mycotoxins in foods. Trends Food Sci. Technol. 46, 252-263 (2015).

3. Martinez, A. W., Phillips, S. T., Butte, M. J. \& Whitesides, G. M. Patterned paper as a platform for inexpensive, low-volume, portable bioassays. Angew. Chem. Int. Ed. Engl. 46, 1318-1320 (2007).

4. Chen, J. J., Shen, C. M. \& Ko, Y. W. Analytical study of a microfludic DNA amplification chip using water cooling effect. Biomed. Microdev. 15, 261-278 (2013).

5. Xu, C. X. \& Yin, X. F. Continuous cell introduction and rapid dynamic lysis for high-throughput single-cell analysis on microfludic chips with hydrodynamic focusing. J. Chromatogr. A 1218, 726-732 (2011)

6. Mery, E. et al. A silicon microfluidic chip integrating an ordered micropillar array separation column and a nano-electrospray emitter for LC/MS analysis of peptides. Sens. Actuator B Chem. 134, 438-446 (2008).

7. Sharma, S. et al. An integrated silicon sensor with microfluidic chip for monitoring potassium and pH. Microfluid. Nanofluidics 10, 1119-1125 (2011).

8. Ge, L. et al. Three-dimensional paper-based electrochemiluminescence immunodevice for multiplexed measurement of biomarkers and point-of-care testing. Biomaterials 33, 1024-1031 (2012).

9. He, Q., Ma, C., Hu, X. \& Chen, H. Method for fabrication of paper-based microfluidic devices by alkylsilane self-assembling and $\mathrm{UV} / \mathrm{O}_{3}$-patterning. Anal. Chem. 85, 1327-1331 (2013).

10. Lu, Y., Shi, W., Qin, J. \& Lin, B. Fabrication and characterization of paper-based microfluidics prepared in nitrocellulose membrane by wax printing. Anal. Chem. 82, 329-335 (2010).

11. Lu, Y., Shi, W., Jiang, L., Qin, J. \& Lin, B. Rapid prototyping of paper-based microfluidics with wax for low-cost, portable bioassay. Electrophoresis $\mathbf{3 0}$, 1497-1500 (2009).

12. Carrilho, E., Martinez, A. W. \& Whitesides, G. M. Understanding wax printing: a simple micropatterning process for paper-based microfluidics. Anal. Chem. 81, 7091-7095 (2009).

13. Apilux, A., Siangproh, W., Praphairaksit, N. \& Chailapakul, O. Simple and rapid colorimetric detection of $\mathrm{Hg}(\mathrm{II})$ by a paper-based device using silver nanoplates. Talanta 97, 388-394 (2012).
14. Bhakta, S. A., Borba, R., Taba, M., Garcia, C. D. \& Carrilho, E. Determination of nitrite in saliva using microfluidic paper-based analytical devices. Anal. Chim. Acta 809, 117-122 (2014).

15. Ortiz-Gomez, l. et al. Tetrazine-based chemistry for nitrite determination in a paper microfluidic device. Talanta 160, 721-728 (2016).

16. Bruzewicz, D. A., Reches, M. \& Whitesides, G. M. Low-cost printing of poly (dimethylsiloxane) barriers to define microchannels in paper. Anal. Chem. 80, 3387-3392 (2008).

17. Abe, K., Suzuki, K. \& Citterio, D. Inkjet-printed microfluidic multianalyte chemical sensing paper. Anal. Chem. 80, 6928-6934 (2008).

18. Abe, K., Kotera, K., Suzuki, K. \& Citterio, D. Inkjet-printed paperfluidic immunochemical sensing device. Anal. Bioanal. Chem. 398, 885-893 (2010).

19. Yamada, K., Henares, T. G., Suzuki, K. \& Citterio, D. Paper-based inkjet-printed microfluidic analytical devices. Angew. Chem. Int. Ed. Engl. 54, 5294-5310 (2015).

20. Chitnis, G., Ding, Z., Chang, C. L., Savran, C. A. \& Ziaie, B. Laser-treated hydrophobic paper: an inexpensive microfluidic platform. Lab Chip 11, 1161-1165 (2011).

21. Cheng, C. M. et al. Millimeter-scale contact printing of aqueous solutions using a stamp made out of paper and tape. Lab Chip 10, 3201-3205 (2010).

22. Curto, V. F. et al. Fast prototyping of paper-based microfluidic devices by contact stamping using indelible ink. RSC Adv. 3, 18811-18816 (2013).

23. Zhang, Y. et al. Equipment-free quantitative measurement for microfluidic paper-based analytical devices fabricated using the principles of movable-type printing. Anal. Chem. 86, 2005-2012 (2014).

24. De Tarso Garcia, P. et al. A handheld stamping process to fabricate microfluidic paper-based analytical devices with chemically modified surface for clinical assays. RSC Adv. 4, 37637-37644 (2014).

25. He, Y., Wu, Y., Xiao, X., Fu, J. \& Xue, G. A low-cost and rapid microfluidic paperbased analytical device fabrication method: flash foam stamp lithography. RSC Adv. 4, 63860-63865 (2014).

26. Yao, X., Jia, T., Xie, C., Fu, J. \& He, Y. Facial fabrication of paper-based flexible electronics with flash foam stamp lithography. Microsyst. Technol. 23 4419-4426 (2017)

27. Lin, Y. et al. Detection of heavy metal by paperbased microfluidics. Biosens. Bioelectron. 83, 256-266 (2016).

28. Xu, W. et al. Superhydrophobic titania nanoparticles for fabrication of paperbased analytical devices: an example of heavy metals assays. Talanta 181, 333-339 (2018).

29. Sadollahkhani, A. et al. Colorimetric disposable paper coated with ZnO@ZnS core-shell nanoparticles for detection of copper ions in aqueous solutions. ACS Appl. Mater. Interfaces 6, 17694-17701 (2014).

30. $L i, J$. J. et al. High efficient adsorption and colorimetric detection of trace copper ions with a functional filter paper. Sens. Actuator B Chem. 223, 853-860 (2016).

31. Fu, Q. et al. A novel fluorescencequenching immunochromatographic sensor for detection of the heavy metal chromium. Biosens. Bioelectron. 49, 399-402 (2013).

32. $\mathrm{Hu}$, J. et al. Paper-based capacitive sensors for identification and quantification chemicals at the point of care. Talanta 165, 419-428 (2017).

33. Kaur, B., Kaur, N. \& Kumar, S. Colorimetric metal ion sensors-a comprehensive review of the years 2011-2016. Coord. Chem. Rev. 358, 12-69. (2018).

34. Cate, D. M., Dungchai, W., Cunningham, J. C., Volckens, J. \& Henry, C. S. Simple, distance-based measurement for paper analytical devices. Lab Chip 13, 2397-2404 (2013)

35. Cate, D. M., Noblitt, S. D., Volckens, J. \& Henry, C. S. Multiplexed paper analytical device for quantification of metals using distance-based detection. Lab Chip 15, 2808-2818 (2015)

36. Pratiwi, R. et al. A selective distance-based paper analytical device for copper(II) determination using a porphyrin derivative. Talanta 174, 493-499 (2017)

37. Alonso-Salces, R. M. et al. A validated solid-liquid extraction method for the HPLC determination of polyphenols in apple tissues: comparison with pressurised liquid extraction. Talanta 65, 654-662 (2005).

38. Teixeira, D. M., Patão, R. F., Coelho, A. V. \& da Costa, C. T. Comparison between sample disruption methods and solid-liquid extraction (SLE) to extract phenolic compounds from Ficus carica leaves. J. Chromatogr. A 1103, 22-28 (2006).

39. Capelo, J. L., Galesio, M. M., Felisberto, G. M., Vaz, C. \& Pessoa, J. C. Micro-focused ultrasonic solid-liquid extraction ( $\mu$ FUSLE) combined with HPLC and fluorescence detection for PAHs determination in sediments: optimization and linking with the analytical minimalism concept. Talanta 66, 1272-1280 (2005). 
40. Gong, Y. et al. A portable and universal upconversion nanoparticle-based lateral flow assay platform for point-of-care testing. Talanta 201, 126-133 (2019).

41. World Health Organization. Guidelines for Drinking-Water Quality 4th edn World Health Organization, 2011). https://www.who.int/water_sanitation_health/ publications/2011/dwq guidelines/en/

42. United States Environmental Protection Agency. Ground Water and Drinking Water (United States Environmental Protection Agency, 2017). https://www. epa.gov/ground-water-and-drinking-water.

43. Guan, Y. F. \& Liu, C. B. Structural parameter analysis and experimental study of micropumps with saw-tooth microchannel. Sens Actuator A 234, 195-205 (2015).

44. Reed, G. F., lynn, F. \& Meade, B. D. Use of coefficient of variation in assessing variability of quantitative assays. Clin. Diagn. Lab Immunol. 9, 1235-1239 (2002).
45. Martinez, A. W., Phillips, S. T., Whitesides, G. M. \& Carrilho, E. Diagnostics for the developing world: microfluidic paper-based analytical devices. Anal. Chem. 82 3-10 (2010).

46. Liu, H. \& Crooks, R. M. Three-dimensional paper microfluidic devices assembled using the principles of origami. J. Am. Chem. Soc. 133, 17564-17566 (2011).

47. Martinez, A. W., Phillips, S. T. \& Whitesides, G. M. Three-dimensional microfluidic devices fabricated in layered paper and tape. Proc. Natl Acad. Sci. USA 105, 19606-19611 (2008)

48. Martinez, A. W. et al. Programmable diagnostic devices made from paper and tape. Lab Chip 10, 2499-2504 (2010).

49. Xiao, L. et al. A rapid, straightforward, and print house compatible mass fabrication method for integrating 3D paper-based microfluidics. Electrophoresis 34, 3003-3007 (2013). 